\title{
A Concise Asymmetric Synthesis of the ADE Fragment of Nakadomarin A
}

\author{
Kateri A. Ahrendt and Robert M. Williams ${ }^{*}$ \\ Department of Chemistry, Colorado State University \\ Fort Collins, CO 80523-1872 \\ rmw@lamar.colostate.edu
}

Supporting Information

General Experimental. Unless otherwise noted, all reagents were obtained from commercial suppliers and were used without further purification. Reactions were carried out in oven-dried glassware under an inert Ar atmosphere. Organic extracts were dried over $\mathrm{Na}_{2} \mathrm{SO}_{4}$ and solvents were removed with a rotary evaporator. Flash column chromatography was carried out using Merck 60 230-400 mesh silica gel. Melting points were determined on a Mel-Temp apparatus and are uncorrected. Optical rotations were measured on a Rudolph Research 589/546-10 polarimeter. IR spectra were recorded on a Nicolet Avatar 320 Fourier Transform infrared spectrometer and only partial data are listed. ${ }^{1} \mathrm{H}$ and ${ }^{13} \mathrm{C}$ NMR spectra were recorded on either a Varian $300 \mathrm{MHz}$ or Varian $400 \mathrm{MHz}$ instrument, as noted. NMR spectra are internally referenced to the residual protio solvent signal. Data for ${ }^{1} \mathrm{H}$ NMR are reported as chemical shift (ppm), multiplicity, coupling constant $(\mathrm{Hz})$, and integration. Data for ${ }^{13} \mathrm{C}$ NMR are reported as chemical shift (ppm). Mass spectra were obtained from the Colorado State University Central Instrumentation Facility. 


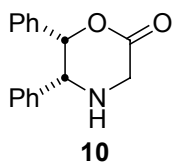

Compound 10. 6-Oxo-2,3-diphenyl-morpholine-4-carboxylic acid tert-butyl ester ${ }^{1}$ (30.0 g, $84.9 \mathrm{mmol}$ ) was suspended in $\mathrm{CH}_{2} \mathrm{Cl}_{2}(425 \mathrm{~mL})$. The reaction flask was placed in a room temperature water bath. A 4M solution of $\mathrm{HCl}$ in dioxane $(106 \mathrm{~mL}, 425$ mmol) was slowly added. The solution was stirred at room temperature for $5 \mathrm{~h}$, until TLC analysis indicated complete removal of the tert-butyl carbamate. The majority of the solvent was then removed in vacuo, and $\mathrm{Et}_{2} \mathrm{O}$ was added to precipitate the hydrochloride salt. The white solid was filtered, rinsed with $\mathrm{Et}_{2} \mathrm{O}$, and dried to provide $24.0 \mathrm{~g}$ (98\% yield) of 5,6-diphenyl-morpholin-2-one hydrochloride. The hydrochloride salt was dissolved in $\mathrm{CH}_{2} \mathrm{Cl}_{2}(300 \mathrm{~mL})$ and sat. $\mathrm{NaHCO}_{3}(5 \mathrm{~mL})$ was slowly added, followed by solid $\mathrm{NaHCO}_{3}(10.0 \mathrm{~g})$. The reaction mixture was stirred for $3 \mathrm{~h}$. Anhydrous $\mathrm{MgSO}_{4}$ was added, the solution was filtered through celite and concentrated to yield $21.5 \mathrm{~g}$ of the title compound as a white solid (89\% yield). Spectral data were consistent with those previously reported ${ }^{1}:{ }^{1} \mathrm{H} \mathrm{NMR}\left(300 \mathrm{MHz}, \mathrm{CDCl}_{3}\right) \delta$ 7.24-7.15 (m, $6 \mathrm{H}), 6.94-6.92(\mathrm{~m}, 2 \mathrm{H}), 6.83-6.82(\mathrm{~m}, 2 \mathrm{H}), 5.69(\mathrm{~d}, J=3.8 \mathrm{~Hz}, 1 \mathrm{H}), 4.64(\mathrm{~d}, J=3.8 \mathrm{~Hz}$, $1 \mathrm{H}), 4.14(\mathrm{~d}, J=18.3,1 \mathrm{H}), 4.03(\mathrm{~d}, J=18.3 \mathrm{~Hz}, 1 \mathrm{H})$.

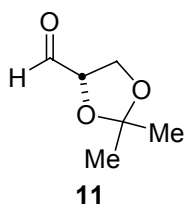

Compound 11. To a solution of 1,2:5,6-di- $O$-isopropylidene-D-mannitol (6.56 g, $25.0 \mathrm{mmol})$ in $\mathrm{CH}_{2} \mathrm{Cl}_{2}(60 \mathrm{~mL})$ was added a solution of saturated $\mathrm{NaHCO}_{3}(3.0 \mathrm{~mL})$. $\mathrm{NaIO}_{4}(8.02 \mathrm{~g}, 37.5 \mathrm{mmol})$ was added slowly, and the suspension was stirred vigorously

\footnotetext{
${ }^{1}$ (a) Aldrich Chemical Co.: (+)-enantiomer, catalog No. 33,181-3 (CAS Registry No. 112741-50-1); (-)-enantiomer, catalog No. 33,184-8 (CAS Registry No. 112741-49-8). (b) Williams, R. M. Aldrichim. Acta 1992, 25, 11. (c) Williams, R. M.; Sinclair, P. J.; DeMong, D. E.; Chen, D.; Zhai, D. Org. Syn. 2002, 80, 18.
} 
for $2 \mathrm{~h}$ until TLC analysis indicated the reaction was complete. Anhydrous $\mathrm{MgSO}_{4}$ was added, and the reaction mixture was filtered and concentrated to provide $5.68 \mathrm{~g}$ of the title compound as a clear oil $(87 \%$ yield). Spectral data were consistent with those previously reported ${ }^{2}: \quad{ }^{1} \mathrm{H}$ NMR $\left(300 \mathrm{MHz}, \mathrm{CDCl}_{3}\right) \delta 9.73(\mathrm{~s}, 1 \mathrm{H}), 4.41-4.37(\mathrm{~m}, 1 \mathrm{H})$, 4.21-4.09 (m, 3H), $1.50(\mathrm{~s}, 3 \mathrm{H}), 1.44(\mathrm{~s}, 3 \mathrm{H})$.

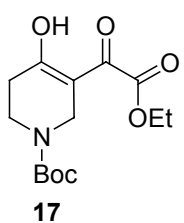

Compound 17. A solution of LiOEt was freshly prepared by the addition of $\mathrm{Li}$ $(1.09 \mathrm{~g}, 157 \mathrm{mmol})$ to anhydrous $\mathrm{EtOH}(140 \mathrm{~mL})$. The solution was cooled to $0{ }^{\circ} \mathrm{C}$, and diethyl oxalate $(22.9 \mathrm{~g}, 157 \mathrm{mmol})$ was added. To this mixture was added $N$-Boc-4piperidinone $(28.5 \mathrm{~g}, 143 \mathrm{mmol})$. The reaction mixture was stirred for $12 \mathrm{~h}$, after which the $\mathrm{EtOH}$ was removed in vacuo. The residue was diluted with $\mathrm{Et}_{2} \mathrm{O}(500 \mathrm{~mL})$, cooled to $0{ }^{\circ} \mathrm{C}$, and $1 \mathrm{~N} \mathrm{HCl}(160 \mathrm{~mL})$ was added slowly (litmus paper indicated acidic solution). The organic layer was extracted, washed with $\mathrm{H}_{2} \mathrm{O}(4 \times 100 \mathrm{~mL})$ and brine $(100 \mathrm{~mL})$. The organic layer was dried, filtered, and concentrated. $\mathrm{SiO}_{2}$ purification $(20 \%$ EtOAc/hex with $1 \% \mathrm{AcOH})$ afforded $21.2 \mathrm{~g}$ of the title compound as an orange oil (50\% yield): IR (film) 2979, 1733, 1699, 1622, 1575, 1418, 1367, 1243, 1165, $1106 \mathrm{~cm}^{-1} ;{ }^{1} \mathrm{H}$ NMR (300 MHz, $\left.\mathrm{CDCl}_{3}\right) \delta 4.46(\mathrm{~s}, 2 \mathrm{H}), 4.37$ (q, $\left.J=7.1,2 \mathrm{H}\right), 3.65$ (t, $\left.J=5.9,2 \mathrm{H}\right), 2.59$ $(\mathrm{t}, J=5.9,2 \mathrm{H}), 2.11(\mathrm{~s}, 1 \mathrm{H}), 1.48(\mathrm{~s}, 9 \mathrm{H}), 1.40(\mathrm{t}, J=7.1,3 \mathrm{H}) ;{ }^{13} \mathrm{C} \mathrm{NMR}(100 \mathrm{MHz}$, $\left.\mathrm{CDCl}_{3}\right) \delta 192.5,173.2,162.3,154.5,107.3,81.3,62.6,41.6,39.2,33.7,28.6,14.3 ;$ FABHRMS calcd. for $\mathrm{C}_{14} \mathrm{H}_{22} \mathrm{~N}_{1} \mathrm{O}_{6}[\mathrm{M}+\mathrm{H}]^{+} 300.1447$, found 300.1434 .

\footnotetext{
${ }^{2}$ Earle, M. J.; Abdur-Rashid, A.; Priestley, N. D. J. Org. Chem. 1996, 61, 5697.
} 


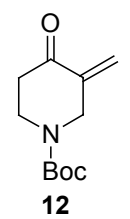

Compound 12. To a mixture of compound $17(10.0 \mathrm{~g}, 33.4 \mathrm{mmol})$ and aqueous formaldehyde $(37 \%, 8.2 \mathrm{~mL}, 100 \mathrm{mmol})$ in THF $(85 \mathrm{~mL})$, a solution of $\mathrm{NaOH}(1.34 \mathrm{~g}$, $33.4 \mathrm{mmol})$ and $\mathrm{H}_{2} \mathrm{O}(5 \mathrm{~mL})$ was added slowly. The reaction mixture was stirred for 20 min, after which TLC analysis indicated no remaining starting material. The reaction mixture was then diluted with $\mathrm{Et}_{2} \mathrm{O}(300 \mathrm{~mL})$ and washed with $\mathrm{H}_{2} \mathrm{O}(2 \times 100 \mathrm{~mL})$ and brine $(50 \mathrm{~mL})$. The organic layer was dried, filtered, and concentrated (water bath temperature $<25^{\circ} \mathrm{C}$ ) to afford the title compound, which was immediately used in the dipolar cycloaddition reaction: ${ }^{1} \mathrm{H}$ NMR $\left(300 \mathrm{MHz}, \mathrm{CDCl}_{3}\right) \delta 6.13(\mathrm{br}, 1 \mathrm{H}), 5.38(\mathrm{br}$, 1H), $4.38(\mathrm{~s}, 2 \mathrm{H}), 3.75(\mathrm{t}, J=6.2 \mathrm{~Hz}, 2 \mathrm{H}), 2.59(\mathrm{t}, J=6.2 \mathrm{~Hz}, 2 \mathrm{H}), 1.47(\mathrm{~s}, 9 \mathrm{H})$.

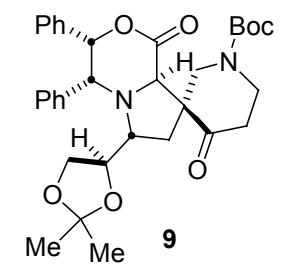

Compound 9. Freshly prepared ene-one $\mathbf{1 2}$ was immediately added to a solution of morpholinone 10 (2.82 g, $11.1 \mathrm{mmol})$ and aldehyde $11(1.52 \mathrm{~g}, 11.7 \mathrm{mmol})$ in toluene (65 mL). The reaction mixture was stirred over $3 \AA$ molecular sieves (previously flame dried under high vacuum) at $50{ }^{\circ} \mathrm{C}$ for $16 \mathrm{~h}$. The reaction mixture was then filtered through celite, concentrated, and purified by $\mathrm{SiO}_{2}$ chromatography (25\% EtOAc/hex) to afford $2.24 \mathrm{~g}$ of the title compound as a white foam (35\% yield): m.p. $88-92{ }^{\circ} \mathrm{C} ;[\alpha]^{23}{ }_{\mathrm{D}}$ $-49.5^{\circ}$ (c 1.0, $\mathrm{CHCl}_{3}$ ); IR (film) 2981, 2932, 1733, 1696, 1477, 1454, 1423, 1367, 1381, $1275,1239,1166,1092,1057,1032,974,856,735,699 \mathrm{~cm}^{-1} ;{ }^{1} \mathrm{H}$ NMR $(500 \mathrm{MHz}$, $\left.\mathrm{CD}_{3} \mathrm{OD}, 50{ }^{\circ} \mathrm{C}\right) \delta$ 7.27-7.22 (m, 3H), 7.16-7.09 (m, 5H), 6.98-6.95 (m, 2H), $6.18(\mathrm{~d}, J=$ 
$3.2 \mathrm{~Hz}, 1 \mathrm{H}), 4.91(\mathrm{~d}, J=3.2 \mathrm{~Hz}, 1 \mathrm{H}), 4.32(\mathrm{~d}, J=13.1 \mathrm{~Hz}, 1 \mathrm{H}), 4.24-4.18(\mathrm{~m}, 1 \mathrm{H}), 4.14$ (s, 1H), 4.03-3.98 (m, 1H), 3.93-3.89 (m, 1H), $3.81(\mathrm{~d}, J=13.1 \mathrm{~Hz}, 1 \mathrm{H}), 3.72-3.68(\mathrm{~m}$, 1H), 3.45-3.39 (m, 1H), 3.35-3.26 (m, 1H), 2.94-2.86 (m, 1H), 2.47-2.42 (m, 1H), 2.20 (dd, $J=6.3,13.0 \mathrm{~Hz}, 1 \mathrm{H}), 1.86(\mathrm{dd}, J=10.6,13.0 \mathrm{~Hz}, 1 \mathrm{H}), 1.50(\mathrm{~s}, 9 \mathrm{H}), 1.44(\mathrm{~s}, 3 \mathrm{H})$, $1.28(\mathrm{~s}, 3 \mathrm{H}) ;{ }^{13} \mathrm{C}$ NMR $\left(100 \mathrm{MHz}, \mathrm{d}_{6}\right.$-DMSO, $\left.100{ }^{\circ} \mathrm{C}\right) \delta 210.0,171.4,154.6,138.5$, $137.5,129.2,128.7,128.3,128.2,127.6,126.7,109.5,79.5,78.9,66.3,64.4,63.9,62.1$, $61.8,52.8,42.9,38.1,36.5,28.7,27.1,25.8 ;$ FAB-HRMS calcd. for $\mathrm{C}_{33} \mathrm{H}_{41} \mathrm{~N}_{2} \mathrm{O}_{7}[\mathrm{M}+\mathrm{H}]^{+}$ 577.2914, found 577.2912.

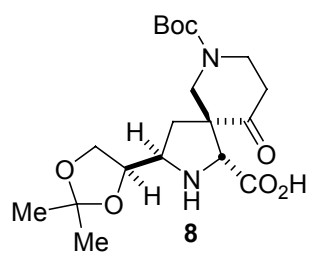

Compound 8. To a solution of compound 9 (3.40 g, $5.90 \mathrm{mmol})$ in EtOH/EtOAc (1:1, $40 \mathrm{~mL}), \mathrm{Pd}(\mathrm{OH})_{2} / \mathrm{C}(830 \mathrm{mg}, 20 \mathrm{wt} \% \mathrm{Pd}$, moist $)$ was added. The reaction flask was flushed with $\mathrm{H}_{2}$, and stirred under a $\mathrm{H}_{2}$ balloon for $16 \mathrm{~h}$, after which it was filtered through celite. The filtrate was concentrated then re-concentrated with toluene to remove residual water from the catalyst. Trituration of the residue with pentane and filtration of the resulting precipitate afforded $2.15 \mathrm{~g}$ of the title compound as a white powder $(91 \%$ yield): m.p. $118-124{ }^{\circ} \mathrm{C} ;[\alpha]^{23}{ }_{\mathrm{D}}-27.5^{\circ}\left(\mathrm{c} 1.0, \mathrm{CHCl}_{3}\right)$; IR (film) $3445,2980,2934,1695$, 1634, 1479, 1423, 1367, 1311, 1245, 1167, 1067, 855, $736 \mathrm{~cm}^{-1} ;{ }^{1} \mathrm{H}$ NMR $(300 \mathrm{MHz}$, $\left.\mathrm{CD}_{3} \mathrm{OD}, 50{ }^{\circ} \mathrm{C}\right) \delta$ 4.29-4.22 (m, 1H), 4.14-3.91 (m, 4H), $3.88(\mathrm{~s}, 1 \mathrm{H}), 3.78-3.69(\mathrm{~m}, 2 \mathrm{H})$, 3.63-3.49 (m, 1H), 2.65-2.61 (m, 2H), 2.47-2.41 (m, 1H), 1.89-1.81 (m, 1H), $1.51(\mathrm{~s}$ 9H), $1.48(\mathrm{~s}, 3 \mathrm{H}), 1.38(\mathrm{~s}, 3 \mathrm{H}) ;{ }^{13} \mathrm{C}$ NMR $\left(100 \mathrm{MHz}, \mathrm{CD}_{3} \mathrm{OD}\right) \delta$ 208.0, 169.9, 155.4, $110.5,80.8,75.6,66.1,65.8,60.3,57.9,50.1,42.0,37.5,27.5,25.6,24.3,22.3$; FABHRMS calcd. for $\mathrm{C}_{19} \mathrm{H}_{31} \mathrm{~N}_{2} \mathrm{O}_{7}[\mathrm{M}+\mathrm{H}]^{+}$399.2131, found 399.2132 . 


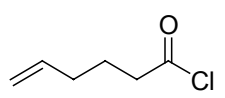

5-Hexenoyl chloride. Catalytic DMF (1 drop) was added to 5-hexeneoic acid (400 mg, $3.50 \mathrm{mmol})$. Oxalyl chloride $(370 \mu \mathrm{L}, 4.20 \mathrm{mmol})$ was added slowly. The reaction mixture was stirred for 15 min after the evolution of gas subsided, then diluted with $\mathrm{Et}_{2} \mathrm{O}$ and filtered. Concentration of the filtrate afforded $380 \mathrm{mg}$ of the title compound as a clear oil (82\%). Spectral data were consistent with those previously reported ${ }^{3}:{ }^{1} \mathrm{H}$ NMR $\left(300 \mathrm{MHz}, \mathrm{CDCl}_{3}\right) \delta$ 5.83-5.69 (m, 1H), 5.10-5.04 (m, 2H), $2.91(\mathrm{t}, J$ $=7.4,2 \mathrm{H}), 2.18-2.11(\mathrm{~m}, 2 \mathrm{H}), 1.88-1.78(\mathrm{~m}, 2 \mathrm{H})$.

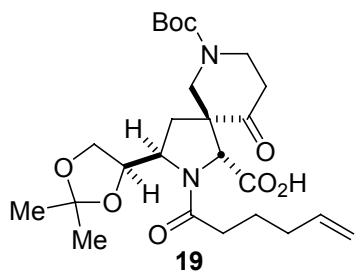

Compound 19. To a solution of compound $8(2.00 \mathrm{~g}, 5.02 \mathrm{mmol})$ in $\mathrm{CH}_{2} \mathrm{Cl}_{2}(10$ $\mathrm{mL})$ was added $\mathrm{NEt}_{3}(2.10 \mathrm{~mL}, 15.1 \mathrm{mmol})$. The solution was stirred for $15 \mathrm{~min}$, then cooled to $0{ }^{\circ} \mathrm{C}$. A solution of 5-hexenoyl chloride (732 mg, $\left.5.52 \mathrm{mmol}\right)$ in $\mathrm{CH}_{2} \mathrm{Cl}_{2}(10$ $\mathrm{mL}$ ) was slowly added. The reaction mixture was stirred at $0{ }^{\circ} \mathrm{C}$ for $1 \mathrm{~h}$, then gradually warmed to room temperature and stirred an additional $1 \mathrm{~h}$. The reaction mixture was then cooled to $0{ }^{\circ} \mathrm{C}$, diluted with $\mathrm{CH}_{2} \mathrm{Cl}_{2}(100 \mathrm{~mL})$, and $1 \mathrm{~N} \mathrm{HCl}(20 \mathrm{~mL})$ was added. The organic layer was extracted, washed with $\mathrm{H}_{2} \mathrm{O}(2 \times 25 \mathrm{~mL})$ and brine $(25 \mathrm{~mL})$. The organic layer was dried, filtered, and concentrated. $\mathrm{SiO}_{2}$ purification afforded $1.87 \mathrm{~g}$ of the title compound as a white foam (75\% yield): m.p. $62-66{ }^{\circ} \mathrm{C} ;[\alpha]^{23}{ }_{\mathrm{D}}+51.8^{\circ}(\mathrm{c} 1.0$, $\mathrm{CHCl}_{3}$ ); IR (film) 3304, 2980, 2935, 1775, 1699, 1668, 1423, 1369, 1264, 1221, 1162 , 1108, 1058, 973, 915, 857, $735 \mathrm{~cm}^{-1} ;{ }^{1} \mathrm{H}$ NMR (400 MHz, d ${ }_{6}$ DMSO, $\left.100{ }^{\circ} \mathrm{C}\right) \delta 5.86-$

\footnotetext{
${ }^{3}$ Cekovik, Z. Tetrahedron Lett. 1972, 13, 749.
} 
$5.75(\mathrm{~m}, 1 \mathrm{H}), 5.04-4.93(\mathrm{~m}, 2 \mathrm{H}), 4.57(\mathrm{~s}, 1 \mathrm{H}), 4.49-4.36(\mathrm{~m}, 1 \mathrm{H}), 4.33-4.20(\mathrm{~m}, 2 \mathrm{H})$ 4.13-4.02 (m, 1H), 4.00-3.94 (m, 1H), 3.65-3.59 (m, 1H), 3.24-3.09 (m, 2H), 2.84-2.69 (m, 1H), 2.65-2.30 (m, 5H), 2.08-2.04 (m, 2H), 1.68-1.59 (m, 2H), $1.45(\mathrm{~s}, 9 \mathrm{H}), 1.38(\mathrm{~s}$, 3H), 1.30 (s, 3H); ${ }^{13} \mathrm{C}$ NMR (100 MHz, $\mathrm{d}_{6}$-DMSO, $\left.100{ }^{\circ} \mathrm{C}\right) \delta 207.2,175.2,171.5,154.6$, $138.9,115.4,109.4,80.5,78.5,66.9,58.8,51.0,42.6,39.7,39.3,34.5,33.2,31.9,28.6$, 26.8, 25.9, 25.8, 24.7; FAB-HRMS calcd. for $\mathrm{C}_{25} \mathrm{H}_{39} \mathrm{~N}_{2} \mathrm{O}_{8}[\mathrm{M}+\mathrm{H}]^{+}$495.2706, found 495.2711.

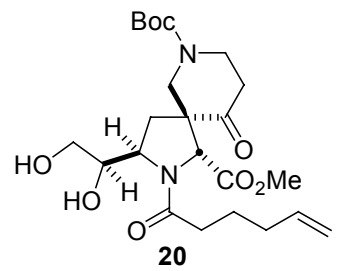

Compound 20. To a solution of compound 19 (88 mg, $0.177 \mathrm{mmol})$ in $\mathrm{MeOH}(1$ $\mathrm{mL}$ ) was added TMS-diazomethane (2.0 M solution in hexanes) until a yellow color persisted. The solution was stirred for 5 min then concentrated. The residue was dissolved in $\mathrm{H}_{2} \mathrm{O}$ saturated $\mathrm{CH}_{3} \mathrm{NO}_{2}(1 \mathrm{~mL})$, and $\mathrm{SnCl}_{2} \cdot 2 \mathrm{H}_{2} \mathrm{O}(40 \mathrm{mg}, 0.177 \mathrm{mmol})$ was slowly added in small portions. The reaction mixture was stirred for $1 \mathrm{~h}$, after which no starting material was observed by TLC. Saturated $\mathrm{NaHCO}_{3}(1 \mathrm{~mL})$ was added and the reaction mixture was diluted with EtOAc $(20 \mathrm{~mL})$. The organic layer was separated, washed with $\mathrm{H}_{2} \mathrm{O}(3 \times 5 \mathrm{~mL})$, then dried, filtered, and concentrated. $\mathrm{SiO}_{2}$ purification $\left(2.5 \% \mathrm{MeOH} / \mathrm{CH}_{2} \mathrm{Cl}_{2}\right)$ afforded $66 \mathrm{mg}$ of the title compound as a colorless amorphous solid (80\% yield); $[\alpha]^{23}{ }_{\mathrm{D}}+7.2^{\circ}\left(c 1.0, \mathrm{CHCl}_{3}\right)$; IR (film) 3399, 2974, 1742, 1695, 1635, $1428,1367,1350,1312,1245,1168,1146,1107,671 \mathrm{~cm}^{-1} ;{ }^{1} \mathrm{H}$ NMR $\left(400 \mathrm{MHz}, \mathrm{d}_{6^{-}}\right.$ DMSO, $\left.100{ }^{\circ} \mathrm{C}\right) \delta 5.86-5.74(\mathrm{~m}, 1 \mathrm{H}), 5.04-4.94(\mathrm{~m}, 2 \mathrm{H}), 4.90-4.83(\mathrm{~m}, 1 \mathrm{H}), 4.58(\mathrm{~s}, 1 \mathrm{H})$, 4.36-4.26 (m, 2H), 4.19-4.12 (m, 1H), 4.09-4.01 (m, 1H), 3.68-3.61 (m, 1H), $3.53(\mathrm{~s}$, 
$3 \mathrm{H}), 3.51-3.39(\mathrm{~m}, 2 \mathrm{H}), 3.39-3.29(\mathrm{~m}, 1 \mathrm{H}), 3.26-3.20(\mathrm{~m}, 1 \mathrm{H}), 2.76-2.66(\mathrm{~m}, 1 \mathrm{H}), 2.65-$ $2.54(\mathrm{~m}, 1 \mathrm{H}), 2.35-2.27(\mathrm{~m}, 1 \mathrm{H}), 2.07-2.00(\mathrm{~m}, 2 \mathrm{H}), 1.77-1.69(\mathrm{~m}, 1 \mathrm{H}), 1.66-1.56(\mathrm{~m}$, 2H), $1.46(\mathrm{~s}, 9 \mathrm{H}) ;{ }^{13} \mathrm{C}$ NMR $\left(100 \mathrm{MHz}, \mathrm{d}_{6}\right.$-DMSO, $\left.100{ }^{\circ} \mathrm{C}\right) \delta 206.0,175.8,170.6,154.7$, $138.9,115.4,80.6,74.8,67.2,67.1,64.8,60.1,59.2,52.4,51.3,51.2,42.8,34.4,33.1$, 28.7, 24.5; FAB-HRMS calcd. for $\mathrm{C}_{23} \mathrm{H}_{37} \mathrm{~N}_{2} \mathrm{O}_{8}[\mathrm{M}+\mathrm{H}]^{+}$469.2550, found 469.2546.

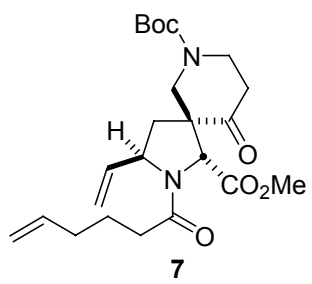

Compound 7. To a solution of compound 20 (400 mg, $0.854 \mathrm{mmol}), \mathrm{PPh}_{3}(896$ $\mathrm{mg}, 3.42 \mathrm{mmol})$, and imidazole $(233 \mathrm{mg}, 3.42 \mathrm{mmol})$ in toluene $(17 \mathrm{~mL})$ at $80{ }^{\circ} \mathrm{C}$ was slowly added $\mathrm{I}_{2}$ in small portions $(650 \mathrm{mg}, 2.56 \mathrm{mmol})$. The reaction mixture was stirred for $2 \mathrm{~h}$ at $80{ }^{\circ} \mathrm{C}$ after the addition of $\mathrm{I}_{2}$ was complete. The reaction mixture was then diluted with $\mathrm{Et}_{2} \mathrm{O}(100 \mathrm{~mL})$ and washed with a solution of $\mathrm{Na}_{2} \mathrm{~S}_{2} \mathrm{O}_{3}(2 \%$ aq, $25 \mathrm{~mL})$. The organic layer was washed with $\mathrm{H}_{2} \mathrm{O}(2 \times 25 \mathrm{~mL})$ and brine $(25 \mathrm{~mL})$. The $\mathrm{Et}_{2} \mathrm{O}$ layer was dried, filtered, and concentrated. $\mathrm{SiO}_{2}$ purification (25\% EtOAc/hex) afforded $223 \mathrm{mg}$ of the title compound as a clear oil (60\% yield): $[\alpha]^{23}{ }_{\mathrm{D}}-9.2^{\circ}\left(c \quad 0.52, \mathrm{CHCl}_{3}\right)$; IR (film) $2976,1743,1700,1652,1423,1367,1350,1279,1239,1199,1268,1145,922 \mathrm{~cm}^{-1} ;{ }^{1} \mathrm{H}$ NMR (400 MHz, $\mathrm{d}_{6}$-DMSO, $\left.100{ }^{\circ} \mathrm{C}\right) \delta 6.00-5.90(\mathrm{~m}, 1 \mathrm{H}), 5.84-5.72(\mathrm{~m}, 1 \mathrm{H}), 5.30-5.19$ (m, 2H), 5.04-4.94 (m, 2H), 4.72-4.63 (m, 1H), $4.64(\mathrm{~s}, 1 \mathrm{H}), 4.35-4.28(\mathrm{~m}, 1 \mathrm{H}), 4.15-$ $4.06(\mathrm{~m}, 1 \mathrm{H}), 3.52(\mathrm{~s}, 3 \mathrm{H}), 3.34-3.21(\mathrm{~m}, 1 \mathrm{H}), 3.14-3.08(\mathrm{~m}, 1 \mathrm{H}), 3.04-2.92(\mathrm{~m}, 1 \mathrm{H})$, 2.66-2.55 (m, 1H), 2.50-2.40 (m, 1H), 2.32-2.23 (m, 2H), 2.07-1.99 (m, 2H), 1.69-1.55 (m, 3H), $1.44(\mathrm{~s}, 9 \mathrm{H}) ;{ }^{13} \mathrm{C}$ NMR $\left(100 \mathrm{MHz}, \mathrm{d}_{6}\right.$-DMSO, $\left.100{ }^{\circ} \mathrm{C}\right) \delta 205.6,173.9,170.3$, 
$154.5,141.1,138.8,116.1,115.5,80.6,66.2,66.1,58.7,52.3,51.0,42.7,36.5,33.6,33.4$, 33.0, 28.6, 24.3; FAB-HRMS calcd. for $\mathrm{C}_{23} \mathrm{H}_{35} \mathrm{~N}_{2} \mathrm{O}_{6}[\mathrm{M}+\mathrm{H}]^{+}$435.2495, found 435.2499.

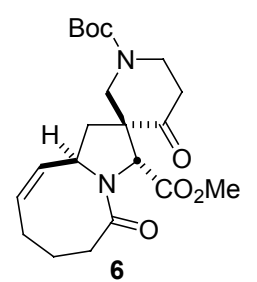

Compound 6. To a solution of compound $7(48.0 \mathrm{mg}, 0.111 \mathrm{mmol})$ in $\mathrm{CH}_{2} \mathrm{Cl}_{2}$ $(100 \mathrm{~mL})$ was added a solution of Grubb's second-generation catalyst $(4.70 \mathrm{mg}, 0.011$ mmol) in $\mathrm{CH}_{2} \mathrm{Cl}_{2}(10 \mathrm{~mL})$. The reaction mixture was refluxed for $2.5 \mathrm{~h}$, upon which TLC analysis indicated the consumption of the starting material. The reaction mixture was cooled to room temperature and the solvent removed in vacuo. The residue was purified by $\mathrm{SiO}_{2}(50 \% \mathrm{EtOAc} / \mathrm{hex})$ to afford $29.0 \mathrm{mg}$ of the title compound as a white solid (65\% yield): m.p. $184-186{ }^{\circ} \mathrm{C} ;[\alpha]^{23}{ }_{\mathrm{D}}-4.9^{\circ}\left(c 0.51, \mathrm{CHCl}_{3}\right)$; IR (film) 2954, 2921, $2849,1753,1698,1655,1650,1461,1422,1366,1277,1241,1166,1145 \mathrm{~cm}^{-1} ;{ }^{1} \mathrm{H}$ NMR $\left(400 \mathrm{MHz}, \mathrm{d}_{6}-\mathrm{DMSO}, 100{ }^{\circ} \mathrm{C}\right) \delta 5.74-5.65(\mathrm{~m}, 1 \mathrm{H}), 5.52-5.47(\mathrm{~m}, 1 \mathrm{H}), 4.88-4.82(\mathrm{~m}$, $1 \mathrm{H}), 4.58(\mathrm{~s}, 1 \mathrm{H}), 4.29-4.23(\mathrm{~m}, 1 \mathrm{H}), 4.13-4.04(\mathrm{~m}, 1 \mathrm{H}), 3.53(\mathrm{~s}, 3 \mathrm{H}), 3.37-3.28(\mathrm{~m}, 1 \mathrm{H})$, 3.18-3.11 (m, 1H), 3.02-2.92 (m, 1H), 2.75-2.48 (m, 4H), 2.19-2.12 (m, 1H), 2.04-1.95 $(\mathrm{m}, 1 \mathrm{H}), 1.87-1.67(\mathrm{~m}, 2 \mathrm{H}), 1.66-1.59(\mathrm{~m}, 1 \mathrm{H}), 1.45(\mathrm{~s}, 9 \mathrm{H}) ;{ }^{13} \mathrm{C}$ NMR $\left(100 \mathrm{MHz}, \mathrm{d}_{6^{-}}\right.$ DMSO, $\left.100{ }^{\circ} \mathrm{C}\right) \delta 204.6,172.2,169.0,153.7,133.5,125.4,79.6,64.9,57.7,56.9,51.0$, 49.4, 41.7, 39.0; 35.3, 31.6, 27.5, 25.1, 22.2; FAB-HRMS calcd. for $\mathrm{C}_{21} \mathrm{H}_{31} \mathrm{~N}_{2} \mathrm{O}_{6}[\mathrm{M}+\mathrm{H}]^{+}$ 407.2182, found 407.2174. 



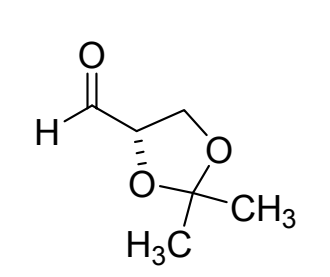

S11 - Compound 11, CDCl3, $300 \mathrm{MHz}$

NMR solvent 


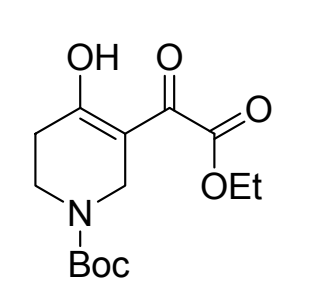

S12 - Compound 17, CDCl3, $300 \mathrm{MHz}$

NMR solvent

$\begin{array}{lllllll}7.5 & 7.0 & 6.5 & 6.0 & 5.5 & 5.0 & 4.5\end{array}$
4.5

4.0

2.0

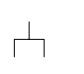

2.1

$\sqcap \sqcap$

0.8

3.5 


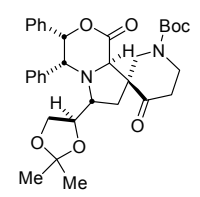

S14 - Compound 9, CD3OD, $500 \mathrm{MHz}, 50$ oC

overlapping peak

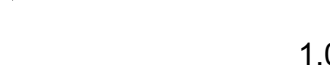




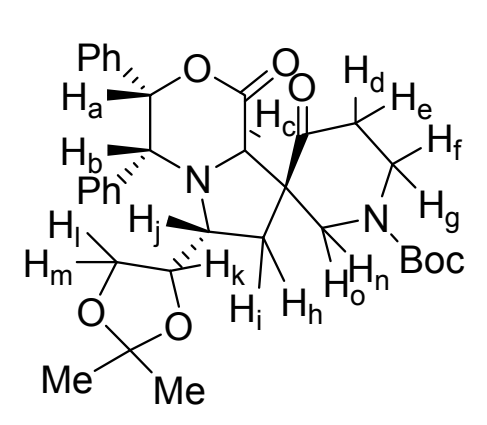

S15 - gCOSY of compound 9 in CD3OD, $500 \mathrm{MHz}, 50$ oC
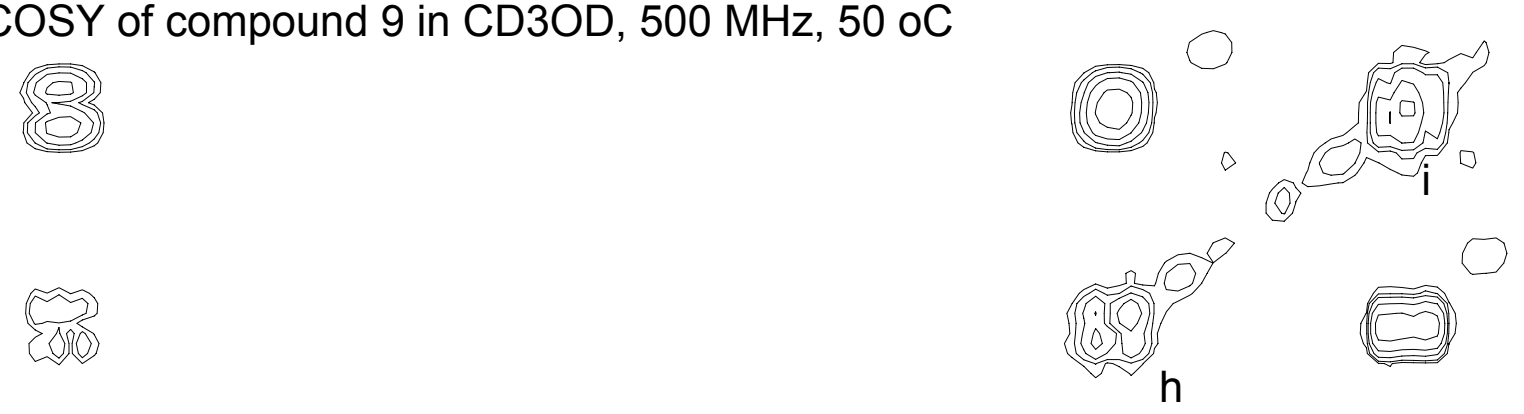

0

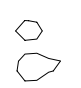
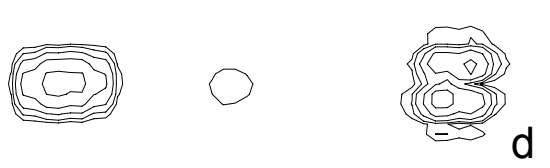

h

4.00

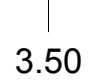

3.00

2.50

2.00

ppm (f2) 


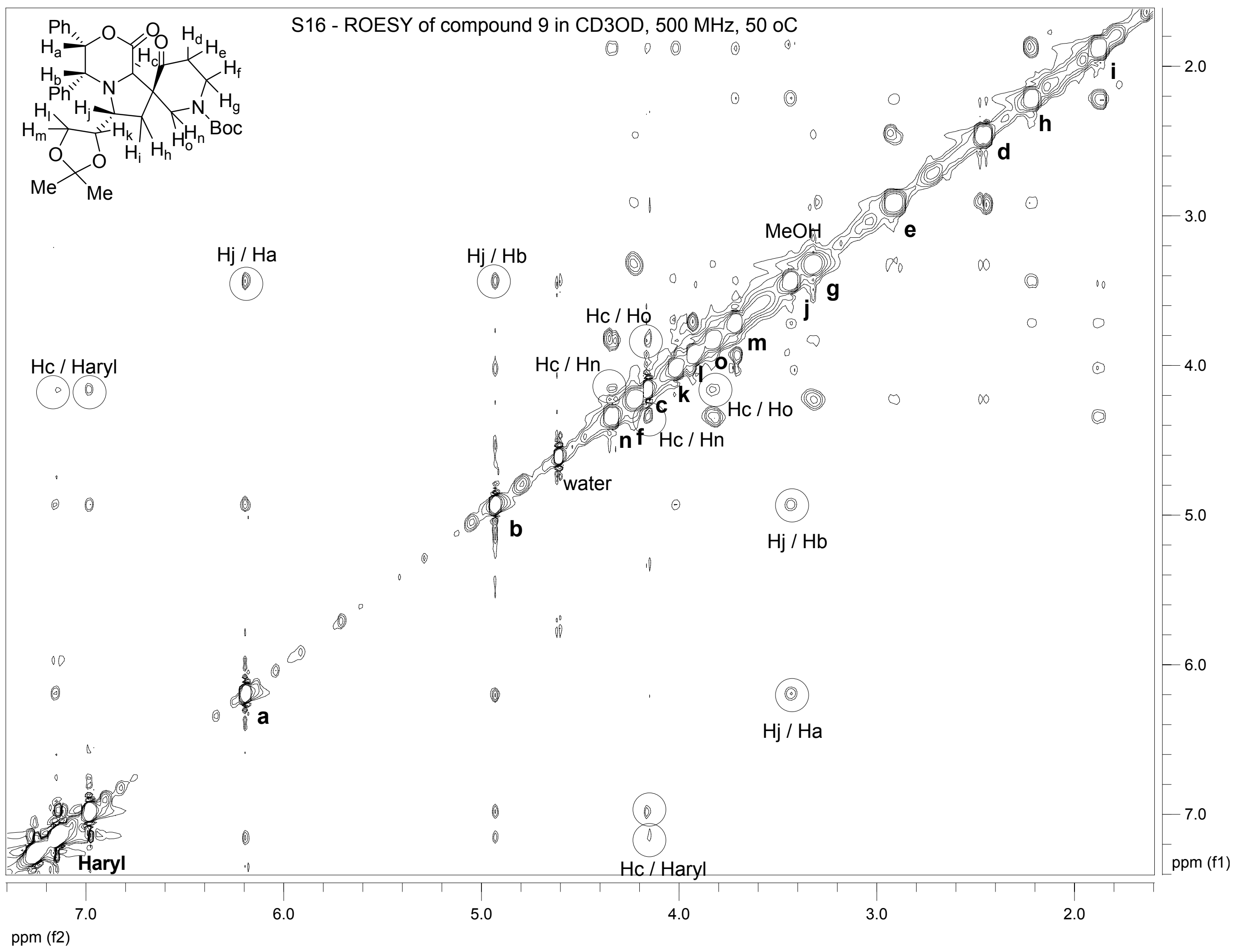





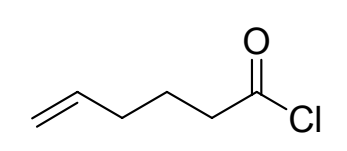

$\mathrm{S} 18-\mathrm{CDCl} 3,300 \mathrm{MHz}$

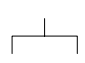

NMR solvent 


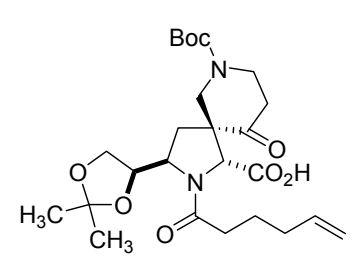

S 19 - Compound 19, d6-DMSO, 100 oC, $400 \mathrm{MHz}$ 


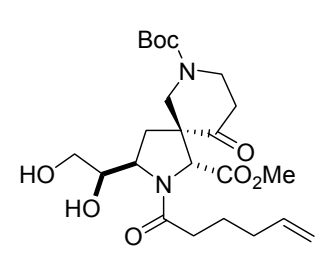

S20 - Compound 20, d6-DMSO, 100 oC, $400 \mathrm{MHz}$

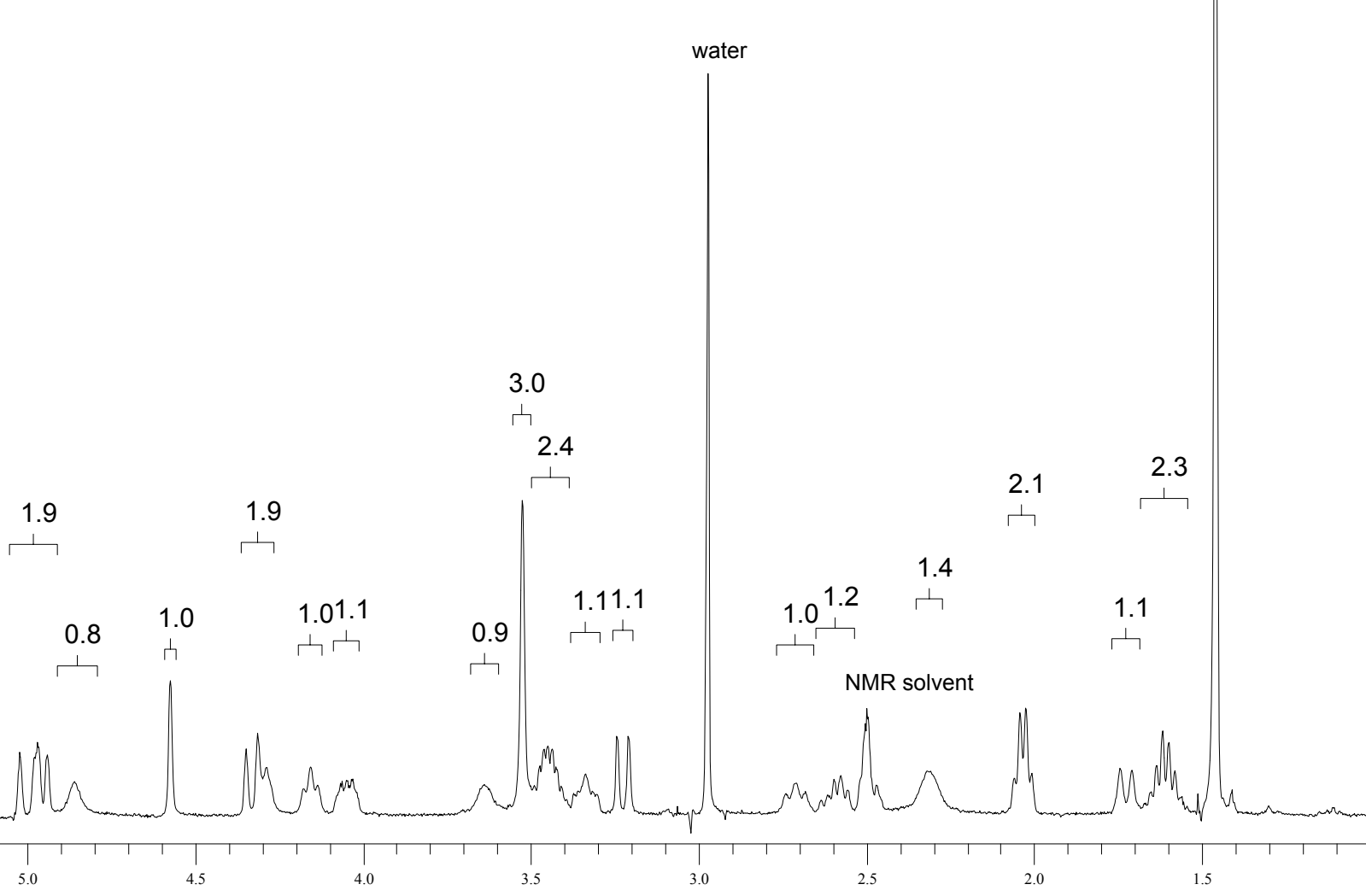




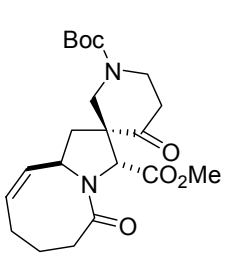

S22 - Compound 6, d6-DMSO, 100 oC, 400 MHz

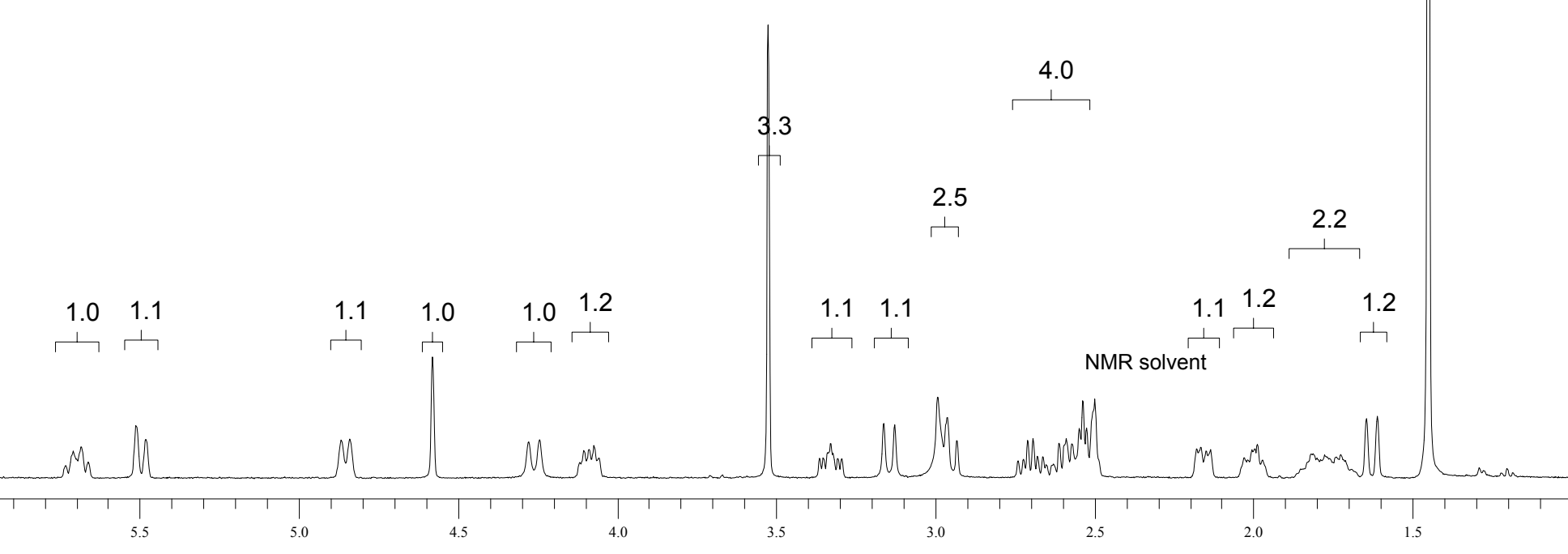

